\title{
Potentiation of the activity of cisplatin in a human colon tumour xenograft model by auristatin PYE, a structural modification of dolastatin 10
}

\author{
EKATHERINE M. PROKOPIOU ${ }^{1}$, PATRICIA A. COOPER ${ }^{1}$, GEORGE R. PETTIT ${ }^{2}$, \\ MICHAEL C. BIBBY ${ }^{1}$ and STEVEN D. SHNYDER ${ }^{1}$ \\ ${ }^{1}$ Institute of Cancer Therapeutics, University of Bradford, Bradford, BD7 1DP, UK; \\ ${ }^{2}$ Department of Chemistry and Biochemistry, Arizona State University, Tempe, AZ 85287-1604, USA
}

Received October 12, 2009; Accepted December 21, 2009

DOI: $10.3892 / \mathrm{mmr} 00000256$

\begin{abstract}
Dolastatin 10, a marine natural product peptide, is now known to act as a vascular disrupting agent (VDA). These VDA properties were not known when other aspects of its promising pre-clinical profile led to initial unsuccessful clinical trials. Auristatin PYE, a synthetic analogue of dolastatin 10, has demonstrated improved activity in preliminary in vivo studies. However, as with other VDAs, tumour eradication was incomplete due to the maintenance of functional vasculature supporting the viable tumour at the periphery of the tumour xenograft, meaning that once the VDA effect subsides, the tumour regrows. One possible strategy for removing this peripheral tumour involves combining VDA therapy with another anticancer drug with a different mechanism of action. Here, we evaluated the effect of combining auristatin PYE with cisplatin in an HCT-116 human colon adenocarcinoma xenograft model. The effects on the growth of subcutaneously implanted HCT-116 xenografts in mice following intraperitoneal administration of a single dose of $4 \mathrm{mgkg}^{-1}$ cisplatin and intravenous administration of $1 \mathrm{mgkg}^{-1}$ auristatin PYE were evaluated compared to the effect of each agent administered alone. The effects on the functional tumour vasculature were also assessed. Statistically significant potentiation $(\mathrm{p}<0.01)$ was noted with a $465 \%$ growth delay for the combination group compared to the control, and 142 and $310 \%$ growth delays for the cisplatin and auristatin PYE groups, respectively. Shutdown of tumour vasculature in the combination group was similar to that observed with auristatin PYE on its own. Auristatin PYE demonstrated synergistic antitumour effects when combined with cisplatin, suggesting that a combination
\end{abstract}

Correspondence to: Dr S.D. Shnyder, Institute of Cancer Therapeutics, University of Bradford, Richmond Road, Bradford BD7 1DP, UK

E-mail: s.d.shnyder@bradford.ac.uk

Key words: auristatin PYE, dolastatin 10, pre-clinical, colon adenocarcinoma, tubulin-binder, combination therapy, cisplatin, vascular disrupting agents chemotherapy regimen would be the most effective strategy when applying this new anticancer drug.

\section{Introduction}

Targeting established tumour vasculature is a very attractive therapeutic strategy for several reasons (1): the chances of drug resistance are less in the genetically more stable endothelial cells than in the tumour cells (2), and drug delivery is unlikely to be compromised. Since the tumour endothelium is readily accessible, shutdown as well as loss of vasculature would have the knock-on effect of starving the nutrient supply, ultimately killing the tumour cells supported (1). Among the different types of vascular disrupting agents (VDAs), to date, small molecule VDAs have progressed the furthest in terms of clinical development. These can be subgrouped as agents which bind to tubulin and result in the depolymerisation of microtubules, leading to the disruption of the endothelial cytoskeleton with the ultimate shutdown of blood flow, and the flavinoids, whose mechanism of action is still unclear. Agents which have progressed to early clinical trials include the tubulin-binders combretastatin A4 phosphate, combretastatin A1 diphosphate, ZD6126, ABT-751, and the flavone-like DMXAA (3-7).

The majority of the tubulin-binding small molecule VDAs are derived from natural products. One which has demonstrated considerable potency as a disruptor of tubulin polymerisation is dolastatin 10, a peptide originally isolated from the Indian Ocean sea hare Dolabella auricularia (8-11). In both in vitro and in vivo pre-clinical studies, dolastatin 10 has demonstrated potent activity against a range of lymphomas, leukaemias and solid tumours (11-13).

Despite these promising pre-clinical results, dolastatin 10 failed to demonstrate any significant clinical activity in Phase II clinical trials (where the protocols were designed without knowledge of the potent VDA activity) in non-small cell lung, prostate, melanoma, colorectal, ovarian, breast and pancreatobiliary tumours $(11,14-20)$. In addition to these results, issues with supply based on a multistep chemical synthesis and lack of water solubility led to the development of analogues (21-23) such as TZT-1027 (auristatin PE), which is currently in Phase II clinical trials (24). 
Another synthetic analogue of dolastatin 10 is auristatin PYE, which differs from auristatin PE in a structural modification from a phenyl to a pyridine (Fig. 1). Following promising preliminary in vitro screening results (25), we carried out more extensive studies (26), which confirmed the activity of the compound through microtubule disruption and its action as a VDA. We also demonstrated that it was more potent than dolastatin 10 in a couple of in vivo human colon xenograft models, thus suggesting that auristatin PYE has good potential as an anticancer drug (26). As with other VDAs, we observed the maintenance of functional vasculature supporting viable tumour at the periphery of the tumour xenografts following treatment. This is a problem as once the VDA effect is removed, the tumour is capable of regrowth. One of the strategies to remove this peripheral tumour is to combine VDA therapy with a standard cytotoxic agent $(1,27,28)$. Thus, we pursued this approach in the present study by evaluating the effect of a combination therapy with auristatin PYE and the standard cytotoxic agent cisplatin in a human colon adenocarcinoma model.

\section{Materials and methods}

Compounds. Auristatin PYE was provided by one of the investigators (G.R.P.) and was initially dissolved in DMSO (Sigma, Poole, UK) and diluted to the appropriate concentration using sterile physiological saline for in vivo studies (10\% DMSO). Cisplatin (Sigma) was dissolved in sterile physiological saline. Compounds were administered as single doses at a $0.1 \mathrm{ml}$ injection volume per $10 \mathrm{~g}$ of body weight, with auristatin PYE administered intravenously and cisplatin intraperitoneally.

Animals. Male Balb/c immunodeficient nude mice (Harlan, Loughborough, UK) aged 6-8 weeks were used. Mice received Harlan 2018 diet (Harlan) and water ad libitum. Mice were kept in cages in an air-conditioned room with regular alternating cycles of light and darkness. All animal procedures were carried out under a project licence issued by the UK Home Office, and UKCCCR guidelines (29) were followed throughout.

Tumour system. HCT-116, a human colon adenocarcinoma cell line (LGC Promochem, Middlesex, UK), was used in the study. Tumours were excised from a donor animal, placed in sterile physiological saline containing antibiotics, and cut into small fragments of $\sim 2 \mathrm{~mm}^{3}$. Under brief general inhalation anaesthesia, fragments were implanted in the left and/or right abdominal flank of each mouse using a trocar. Once the tumours could accurately be measured using callipers (mean tumour volume of $\sim 32 \mathrm{~mm}^{3}$ ), the mice were allocated into groups of 11 (8 mice for chemotherapy studies, 3 for vascular shutdown study) by restricted randomisation to keep group mean tumour size variation to a minimum.

Chemotherapy studies. Compounds were administered by single injection, with the day of therapy designated as day 0 . Both compounds were administered at doses below their previously established maximum tolerated doses (MTDs), namely $2 \mathrm{mgkg}^{-1}$ for auristatin PYE and $10 \mathrm{mgkg}^{-1}$ for cisplatin $(27,28)$. Therefore, auristatin PYE was administered at $1 \mathrm{mgkg}^{-1}$ and cisplatin at $4 \mathrm{mgkg}^{-1}$. For the combination protocol group,

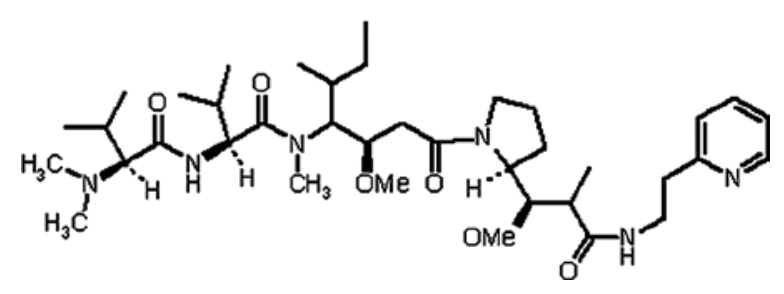

Figure 1. Structure of auristatin PYE.

auristatin PYE was administered 20 min following cisplatin based on previous combination studies with VDAs $(27,28)$. The control group remained untreated. The effects of therapy were assessed as previously described (28). Briefly, daily 2-dimensional caliper measurements of the tumours were taken, with volumes calculated using the formula: $\left(a^{2} \times b\right) / 2$, where $a$ is the smaller and $b$ the larger diameter of the tumour. Tumour volume was then normalised to the respective volume on day 0 , and semi-log plots of relative tumour volume vs. time were made. Mann-Whitney $U$ tests were performed to determine the statistical significance of any differences in growth rate (based on tumour volume doubling time) between control and treated groups, and between the two compounds.

Vascular shutdown. In order to assess the effects of treatment with auristatin PYE alone or in combination with cisplatin on the functional vasculature of the HCT-116 tumours, groups of tumour-bearing mice $(n=3)$ were set up as described above. Once tumours had reached a volume of $\sim 150 \mathrm{~mm}^{3}$, to ensure that an established tumour vascular network was in place, mice were treated with the same regimens as those used for the chemotherapy studies above. Hoechst 33342 (bisbenzimide; Sigma) was used to assess the functional tumour vasculature $(30,31)$. Previously, it was shown that 6 -h post-treatment is the optimum time at which to demonstrate vascular shutdown with auristatin PYE (26). Thus, this was the time period used here. Hoechst 33342 was dissolved in sterile saline and injected intravenously by the tail vein at $40 \mathrm{mgkg}^{-1}$. One minute after injection, the mice were sacrificed by cervical dislocation, and the tumours were carefully and rapidly excised. The tumour from each mouse was wrapped in aluminium foil and immediately immersed in liquid nitrogen and stored at $-80^{\circ} \mathrm{C}$ until ready for ultracryotomy.

Frozen sections $(10 \mu \mathrm{m})$ were obtained at $\sim 100-\mu \mathrm{m}$ intervals through the tumour. Five random fields from each of five random sections were examined for each tumour under UV illumination using a Leica DMRB microscope, with images captured digitally through a JVC 3-CCD camera and processed using AcQuis software (Synoptics, Cambridge, UK). The functional vasculature was assessed by placing a $22 \times 16$ grid over the captured digital image and counting the number of points on the grid which overlaid the fluorescently stained cells. Comparisons were made between the percentage of vasculature in the control and treated tumours.

\section{Results}

Chemotherapy studies. For all of the treatments administered, observed toxicity was negligible within the parameters laid 
Table I. In vivo evaluation of a combination of auristatin PYE and cisplatin against HCT-116 tumours.

\begin{tabular}{|c|c|c|c|c|}
\hline \multicolumn{2}{|c|}{ Dose $\left(\mathrm{mgkg}^{-1}\right)$} & \multirow[t]{2}{*}{ Mean RTV2a (days) } & \multirow[t]{2}{*}{ Growth delay (days) } & \multirow[t]{2}{*}{ Significance } \\
\hline Auristatin PYE & Cisplatin & & & \\
\hline- & - & 3.1 & - & - \\
\hline 1 & - & 9.6 & 6.7 & $\mathrm{p}<0.01$ \\
\hline- & 4 & 4.4 & 1.3 & NS \\
\hline 1 & 4 & 14.5 & 11.5 & $\mathrm{p}<0.01$ \\
\hline
\end{tabular}

${ }^{a}$ Mean tumour volume doubling-time. RTV, relative tumour volume; NS, not significant.

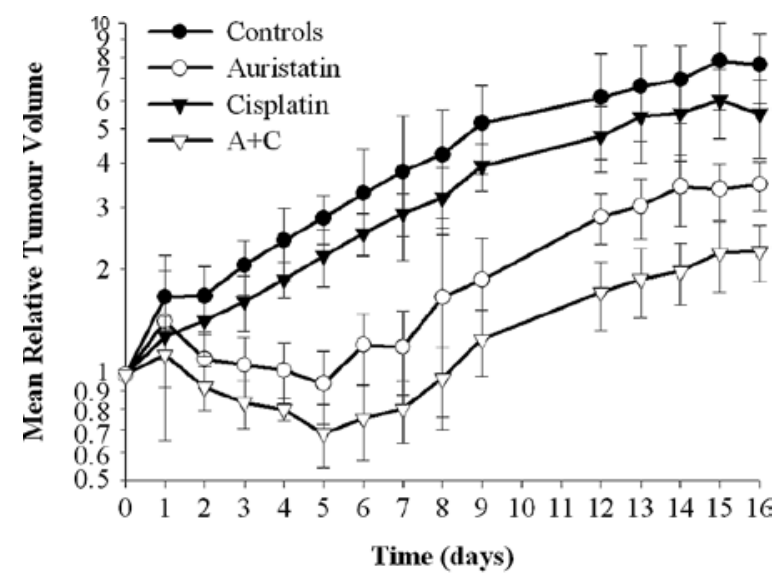

Figure 2. Evaluation of the efficacy of auristatin PYE and cisplatin administered either individually at doses below their respective MTDs, or in combination (with auristatin PYE administered 20 min after cisplatin administration) in an HCT-116 colon adenocarcinoma subcutaneous xenograft model. Data points represent the mean $\pm \operatorname{SD}(n=8)$.
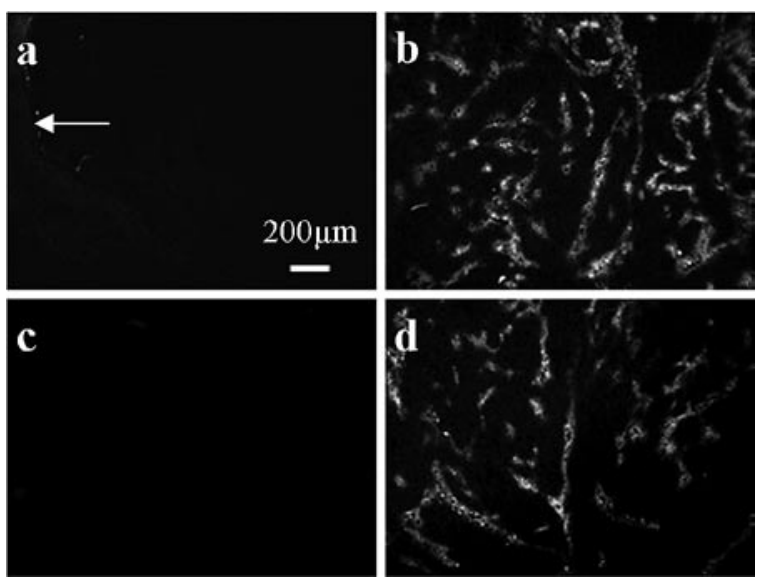

Figure 3. Images of Hoechst 33342-stained functional vascular elements in cryosections of HCT-116 tumours treated with either auristatin PYE at $1 \mathrm{mgkg}^{-1}$ (a), cisplatin at $4 \mathrm{mgkg}^{-1}$ (b), or a combination of cisplatin at $4 \mathrm{mgkg}^{-1}$ followed by auristatin PYE (c). Untreated control vasculature is seen in (d). In (a), functional vasculature is apparent at the tumour periphery (arrow). Samples were taken $6 \mathrm{~h}$ following treatment.

out within the UKCCCR guidelines (29). Auristatin PYE demonstrated a statistically significant tumour growth delay when administered alone, while no significant delay was noted

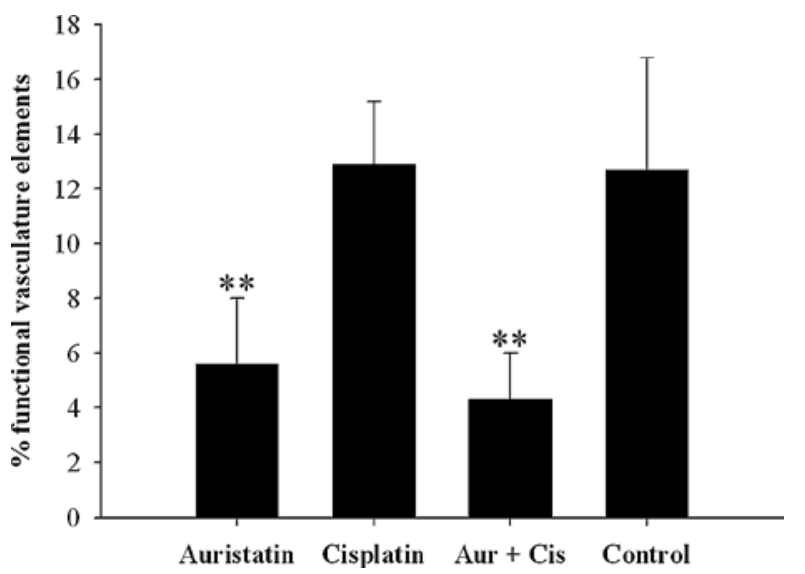

Figure 4. Quantitation of functional tumour vasculature $6 \mathrm{~h}$ following administration of auristatin PYE and/or cisplatin. A statistically significant reduction in functional tumour vasculature $\left({ }^{* *} \mathrm{p}<0.01\right)$ was noted in groups containing auristatin PYE in their treatment regimen.

for cisplatin administered at $4 \mathrm{mgkg}^{-1}$. In combining the two agents, the growth delay was highly significant, with almost a doubling of the delay noted for auristatin PYE alone, and was much greater than would be expected from the summation of the growth delays of the two agents administered separately. This suggests that the effects observed were synergistic rather than just additive (Fig. 2 and Table I). The combination did not result in any significant weight loss compared to the agents administered alone.

Vascular shutdown assessment. Clearly the amount of functional vasculature (as determined by the incorporation of Hoechst 33342 dye into the nuclei of functioning endothelial cells) was decreased in the sections from the groups which included auristatin PYE in their treatment protocols (Fig. 3). This was confirmed by quantitative analysis, where the functional vasculature was significantly reduced to a similar extent $(\mathrm{p}<0.01)$ in both the groups treated with auristatin PYE and the combination protocol, compared to the untreated control and cisplatin groups (Fig. 4).

\section{Discussion}

Vascular disrupting agents have shown much promise preclinically as anticancer therapeutics, and a small number are 
currently being investigated in clinical trials. However, the failure of such agents to target the peripheral tumour rim means that their efficacy as a single-agent therapeutic strategy is in need of improvement (32). One revised strategy involves treatment in combination with radiotherapy or a standard chemotherapeutic agent that can destroy the remaining tumour cells. There have been several pre-clinical studies that demonstrated improved efficacy (33). This approach has recently been reported as having some success in Phase II clinical trials using combretastatin A4 phosphate in combination with carboplatin and paclitaxel (34).

Auristatin PYE, a synthetic analogue of dolastatin 10, offers several advantages over dolastatin 10 that may afford it a better chance of success in the clinic. These advantages include improved solubility, an easier route to synthesis $(25,35)$, and better tolerance when administered in vivo (26). We previously demonstrated that auristatin PYE exhibits improved activity compared with dolastatin 10 in pre-clinical studies in human colon adenocarcinoma xenograft models when administered as a single agent. Such activity can be attributed to the tumour vasculature-targeting effects of auristatin PYE (26). The key aim of this study was to ascertain whether the antivascular effects of auristatin PYE could be exploited in combination therapy to improve the efficacy of the standard agent cisplatin in the HCT-116 colon adenocarcinoma xenograft model.

Significant potentiation of the effect of cisplatin was noted. The growth delay for the combined treatment was greater than the sum of the growth delays noted for auristatin PYE and cisplatin alone. These findings demonstrated that a synergistic, rather than simply an additive, effect was achieved with combination therapy. The fact that both drugs were administered at doses lower than their MTDs, without any discernible toxicity, gives considerable scope for optimising the treatment protocol, either by repeat dosing or changing the dose administered. In addition, altering the sequence and time interval between the administration of auristatin PYE and cisplatin may be a key factor, which has proved beneficial in combination therapies $(32,36)$.

While a clear improvement was noted in terms of efficacy for the combination drug treatment, evaluation of vascular shutdown showed no significant increase in the combination group compared to auristatin PYE administered alone. This has been previously demonstrated in other VDA combination studies (27). Although cisplatin had a negligible effect on the vasculature itself, the increased tumour growth delay was due to vascular shutdown, leading to cisplatin being retained in the tumour for longer periods and thus enabling it to have a greater effect on the tumour cells. The consequence of this was a prolonged growth delay.

In summary, the therapeutic strategy of administering auristatin PYE at a sub-MTD dose in combination with another anticancer drug having a different mechanism of action warrants further investigation as a way of enhancing the potential of auristatin PYE in future human cancer clinical trials.

\section{Acknowledgements}

Very necessary financial assistance was received from grants RO1 CA 90441-03-05, 2R56 CA 09441-06A1 and 5RO1 CA 090441-07 of the Division of Cancer Treatment and
Diagnosis, NCI, DHHS, and the Arizona Biomedical Research Commission (G.R. Pettit).

\section{References}

1. Hinnen $P$ and Eskens FA: Vascular disrupting agents in clinical development. Br J Cancer 96: 1159-1165, 2007.

2. Kerbel R and Folkman J: Clinical translation of angiogenesis inhibitors. Nat Rev Cancer 2: 727-739, 2002.

3. Patterson DM, Charnley N, Saleem A, et al: Phase I evaluation of vascular disrupting agent OXi4503. J Clin Oncol 26: 3551, 2008.

4. Cooney MM, Savvides P, Agarwala S, et al: Phase II study of combretastatin A4 phosphate (CA4P) in patients with advanced anaplastic thyroid carcinoma (ATC). J Clin Oncol 24: 5580, 2006.

5. LoRusso PM, Gadgeel SM, Wozniak A, et al: Phase I clinical evaluation of ZD6126, a novel vascular-targeting agent, in patients with solid tumors. Invest New Drugs 26: 159-167, 2008.

6. Mauer AM, Cohen EE, Ma PC, et al: A phase II study of ABT-751 in patients with advanced non-small cell lung cancer. J Thorac Oncol 3: 631-636, 2008.

7. Li J, Jameson MB, Baguley BC, Pili R and Baker SD: Population pharmacokinetic-pharmacodynamic model of the vasculardisrupting agent 5,6-dimethylxanthenone-4-acetic acid in cancer patients. Clin Cancer Res 14: 2102-2110, 2008.

8. Bai R, Roach MC, Jayaram SK, et al: Differential effects of active isomers, segments and analogs of dolastatin 10 on ligand interactions with tubulin. Correlation with cytotoxicity. Biochem Pharmacol 45: 1503-1515, 1993.

9. Bai RL, Pettit GR and Hamel E: Binding of dolastatin 10 to tubulin at a distinct site for peptide antimitotic agents near the exchangeable nucleotide and vinca alkaloid sites. J Biol Chem 265: 17141-17149, 1990.

10. Pettit GR, Kamano Y, Herald CL, et al: The isolation and structure of a remarkable murine animal antineoplastic constituent: dolastatin 10. J Am Chem Soc 109: 6883-6885, 1987.

11. Pettit GR: Dolastatin anticancer drugs. In: Marine Anticancer Compounds in the Era of Targeted Therapies. Chabner B (ed). Permanyer Publications, Barcelona, pp19-50, 2009.

12. Kalemkerian GP, Ou X, Adil MR, et al: Activity of dolastatin 10 against small-cell lung cancer in vitro and in vivo: induction of apoptosis and bcl-2 modification. Cancer Chemother Pharmacol 43: 507-515, 1999.

13. Mohammad RM, Pettit GR, Almatchy VP, Wall N, Varterasian M and Al-Katib A: Synergistic interaction of selected marine animal anticancer drugs against human diffuse large cell lymphoma. Anticancer Drugs 9: 149-156, 1998.

14. Krug LM, Miller VA, Kalemkerian GP, et al: Phase II study of dolastatin-10 in patients with advanced non-small cell lung cancer. Ann Oncol 11: 227-228, 2000.

15. Vaishampayan U, Glode M, Du W, et al: Phase II study of dolastatin-10 in patients with hormone-refractory metastatic prostate adenocarcinoma. Clin Cancer Res 6: 4205-4208, 2000.

16. Margolin K, Longmate J, Synold TW, et al: Dolastatin-10 in metastatic melanoma: a phase II and pharmokinetic trial of the California Cancer Consortium. Invest New Drugs 19: 335-340, 2001.

17. Saad ED, Kraut EH, Hoff PM, et al: Phase II study of dolastatin-10 as first-line treatment for advanced colorectal cancer. Am J Clin Oncol 25: 451-453, 2002.

18. Hoffman MA, Blessing JA and Lentz SS: A phase II trial of dolastatin-10 in recurrent platinum-sensitive ovarian carcinoma: a Gynecologic Oncology Group Study. Gynecol Oncol 89: 95-98, 2003.

19. Perez EA, Hillman DW, Fishkin PA, et al: Phase II trial of dolastatin-10 in patients with advanced breast cancer. Invest New Drugs 23: 257-261, 2005.

20. Kindler HL, Tothy PK, Wolff R, et al: Phase II trials of dolastatin-10 in advanced pancreaticobiliary cancers. Invest New Drugs 23: 489-493, 2005.

21. Pettit GR, Srirangam JK, Barkoczy J, et al: Antineoplastic agents 337. Synthesis of dolastatin 10 structural modifications. Anticancer Drug Des 10: 529-544, 1995.

22. Miyazaki K, Kobayashi M, Natsume T, et al: Synthesis and antitumor activity of novel dolastatin 10 analogs. Chem Pharm Bull 43: 1706-1718, 1995.

23. Harrigan GG, Luesch H, Yoshida WY, et al: Symplostatin 1: a dolastatin 10 analogue from the marine cyanobacterium Symploca hydnoides. J Nat Prod 61: 1075-1077, 1998. 
24. Riely GJ, Gadgeel S, Rothman I, et al: A phase 2 study of TZT-1027, administered weekly to patients with advanced non-small cell lung cancer following treatment with platinumbased chemotherapy. Lung Cancer 55: 181-185, 2007.

25. Fennell BJ, Carolan S, Pettit GR and Bell A: Effects of the antimitotic natural product dolastatin 10 and related peptides on the human malarial parasite Plasmodium falciparum. J Antimicrob Chemother 51: 833-841, 2003.

26. Shnyder SD, Cooper PA, Millington NJ, Pettit GR and Bibby MC: Auristatin PYE, a novel synthetic derivative of dolastatin 10, is highly effective in human colon tumour models. Int J Oncol 31: 353-360, 2007.

27. Shnyder SD, Cooper PA, Gyselinck N, Hill BT, Double JA and Bibby MC: Vinflunine potentiates the activity of cisplatin but not 5-fluorouracil in a transplantable murine adenocarcinoma model. Anticancer Res 23: 4815-4820, 2003.

28. Shnyder SD, Cooper PA, Pettit GR, Lippert JW III and Bibby MC: Combretastatin A-1 phosphate potentiates the antitumour activity of cisplatin in a murine adenocarcinoma model. Anticancer Res 23: 1619-1623, 2003.

29. Workman P, Twentyman P, Balkwill F, et al: United Kingdom Co-ordinating Committee on Cancer Research (UKCCCR) Guidelines for the Welfare of Animals in Experimental Neoplasia (2nd edition). Br J Cancer 77: 1-10, 1998.
30. Quinn PK, Bibby MC, Cox JA and Crawford SM: The influence of hydralazine on the vasculature, blood perfusion and chemosensitivity of MAC tumours. Br J Cancer 66: 323-330, 1992.

31. Smith KA, Hill SA, Begg AC and Denekamp J: Validation of the fluorescent dye Hoechst 33342 as a vascular space marker in tumours. Br J Cancer 57: 247-253, 1988.

32. Kanthou $\mathrm{C}$ and Tozer GM: Microtubule depolymerizing vascular disrupting agents: novel therapeutic agents for oncology and other pathologies. Int J Exp Pathol 90: 284-294, 2009.

33. Horsman MR and Siemann DW: Pathophysiologic effects of vascular-targeting agents and the implications for combination with conventional therapies. Cancer Res 66: 11520-11539, 2006.

34. Zweifel M, Jayson G, Reed N, et al: Combretastatin A-4 phosphate (CA4P) carboplatin and paclitaxel in patients with platinum-resistant ovarian cancer: Final phase II trial results. J Clin Oncol 27: 5502, 2009.

35. Amador ML, Jimeno J, Paz-Ares L, Cortes-Funes $\mathrm{H}$ and Hidalgo M: Progress in the development and acquisition of anticancer agents from marine sources. Ann Oncol 14: 1607-1615, 2003.

36. Siemann DW and Horsman MR: Vascular targeted therapies in oncology. Cell Tissue Res 335: 241-248, 2009. 